\title{
Analysis of the spectral response of fractal antennas related with its geometry and current paths
}

\author{
Alexander Cuadrado ${ }^{a, b}$, José M. López-Alonso ${ }^{a}$, Juan C. Martínez- Antón ${ }^{a}$, \\ José M. Ezquerro ${ }^{a}$, Francisco J. González ${ }^{c}$, and Javier Alda ${ }^{a}$ \\ ${ }^{a}$ Applied Optics Complutense Group, University Complutense of Madrid, \\ Ave. Arcos de Jalón, 118. 28037 Madrid. Spain \\ ${ }^{b}$ Laser Processing Group. Institute of Optics. Consejo Superior de Investigaciones Científicas \\ Serrano 121. 28006 Madrid. Spain. \\ ${ }^{c}$ Coordinación para la Innovación y la Aplicación de la Ciencia y la Tecnología, Universidad \\ Autónoma de San Luis Potosí, Sierra Leona 550, Lomas 2a Sección, SLP, Mexico
}

\begin{abstract}
Fractal antennas have been proposed to improve the bandwidth of resonant structures and optical antennas. Their multiband characteristics are of interest in radiofrequency and microwave technologies. In this contribution we link the geometry of the current paths built-in the fractal antenna with the spectral response. We have seen that the actual currents flowing through the structure are not limited to the portion of the fractal that should be geometrically linked with the signal. This fact strongly depends on the design of the fractal and how the different scales are arranged within the antenna. Some ideas involving materials that could actively respond to the incoming radiation could be of help to spectrally select the response of the multiband design.
\end{abstract}

Keywords: optical antennas, fractal antennas, multiband antennas

\section{INTRODUCTION}

The use of resonant metallic structures coupled to opto-electronic transducers has made possible the appearance of antenna-coupled devices, or optical antennas. ${ }^{1-3}$ Optical antennas benefit from the tiny size of the transducer, and the selective properties of antennas and resonant elements. The small volume of the transducer makes fast devices, even when involving thermal mechanisms. Three types of physical phenomena have been used so far to generate an electrical signal readable by an external circuit: rectification through a metal-oxide-metal junction, ${ }^{4}$ bolometric variation of the ressistivity of the element, ${ }^{5}$ and thermo-electric transduction. ${ }^{6}$ Most of these mechanism are directly related with the spatial distribution of electric field, $\vec{E}$, within the structure. The currents built in the antenna are proportional to this electric field through the Ohm's law, $\vec{J}=\sigma \vec{E}$, where $\sigma$ represents the electric conductivity, and $\vec{J}$ is the current density vector. Metal-oxide-metal junctions rectify these currents and produce the signal. However, both bolometric and thermoelectric systems are dependent on the heat conversion of induced currents as Joule heat. This contribution is proportional to $\sigma|\vec{E}|^{2}$. Therefore, an special attention should be paid to the evaluation of the electric field within the antenna structure, both for the bolometric and thermoelectric transducer, and for the metal-oxide-metal rectifiers. It should be noted that electrical and optical constants are frequency dependent within the optical range (including the infrared), and metals do not behave as perfect conductors anymore. ${ }^{7}$ On the other hand, the transduction mechanism involved in the detection of light imposes some constrains about the auxiliary elements around the resonant geometries. ${ }^{8,9}$

In this paper we rely on computational electromagnetism simulation packages as Comsol Multihphysis. We have proved its capability to predict and analyze the electromagnetic and thermal behavior of nano-antennas in a variety of configurations. ${ }^{10}$

Further author information: (Send correspondence to Javier Alda)

Javier Alda: E-mail: javier.alda@ucm.es, Telephone: +34.91.3946874

Plasmonics: Metallic Nanostructures and Their Optical Properties XIII, edited by Allan D. Boardman, Din Ping Tsai, Proc. of SPIE Vol. 9547, 954736 - @ 2015 SPIE · CCC code: 0277-786X/15/\$18 · doi: 10.1117/12.2187946 
When considering the spectral response of an optical antenna, its selectivity properties are revealed from the calculations. Although there exist wide bandwidth designs, we have chosen here a different approach to propose a multiband antenna structure. Compound geometries combining orthogonal dipoles have been suggested for the analysis of the state of polarization of an incoming beam,. In this contribution we use them as elements able to respond within a wider band spectrum. In this case, this capability is related to a polarization selectivity. To expand these properties, fractal antennas are proposed as good candidates to resonate at different frequencies according with the fractal geometry configuration. Also, fractal resonant structures have been used to modify spectral distributions as it happens with frequency selective surface, or metamaterials. ${ }^{11}$ These fractal resonant structures have been also proposed as focusing elements beyond diffraction limits. ${ }^{12}$ In this contribution the compound and fractal geometries are analyzed in subsections 2.1 and 2.2. Our main objective is to understand their behavior as detectors. This is why we focus on the analysis of fhe spatial distribution of the dissipated power and electric field, and how they are related with the spectral response of these geometries. Finally, in section 3 we summarize the main conclusions of this contribution.

\section{SPECTRAL RESPONSE AND FRACTAL GEOMETRY}

The geometry of the antenna determines the spectral and polarization response of antenna-coupled detectors. A feasible goal is to generate a simple geometry able to resonate within an extended band wider than, for example, the bandwidth of dipole antennas. In this section, we first explore the spectral behavior of crossed dipoles having different lengths. These compound geometries respond with a wider bandwidth for selected polarization states. Then, fractal geometries are analyzed to produce an actual multispectral response related with the different portions of the self-similar geometries.

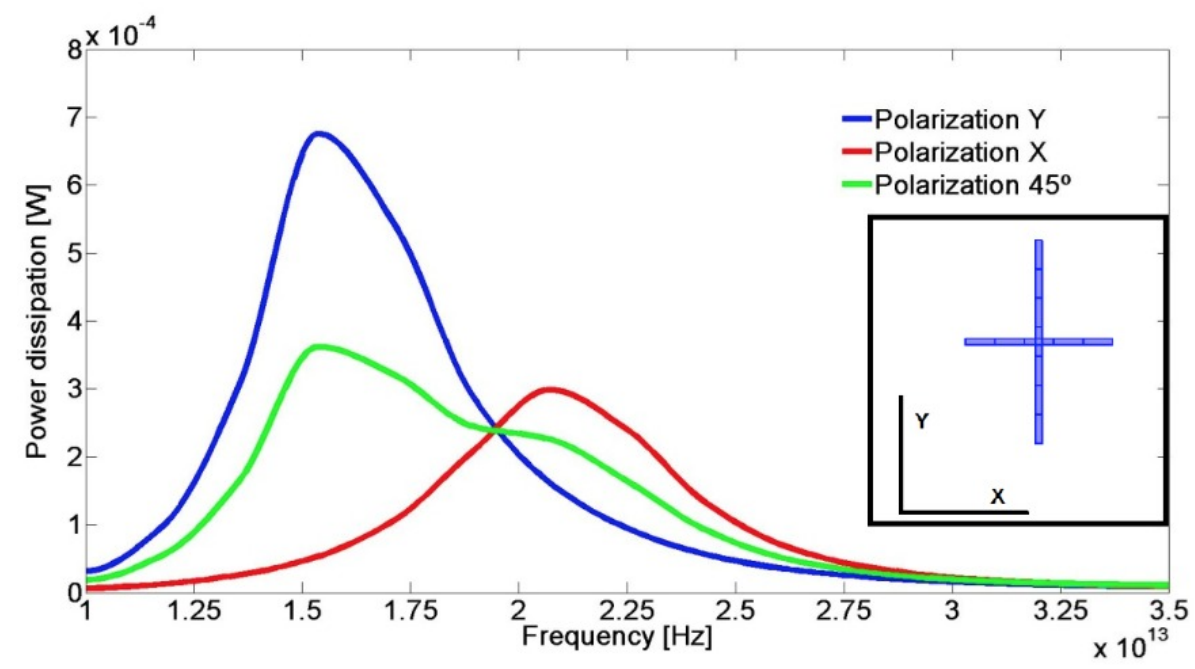

Figure 1. Spectral response of the crossed dipole for three different polarization states: $0^{\circ}, 45 \circ$ and 90 . The parameter of interest is the power dissipated by the whole structure. This structure should be considered as a distributed bolometer. The inset shows the geometry of the antenna.

\subsection{Compound geometries}

The association of two dipoles has been proposed for polarization multiplexing ${ }^{13}$ and for Stokes' parameter detection. ${ }^{6,14}$ However, when dipoles are different in length, their resonances are located at different wavelengths. At the same time, when considering optical frequencies, the resonant length predicted for perfect conductors does not apply and the actual length of the dipole has to be properly scaled. ${ }^{7}$

The proposed design contains two crossed dipoles with lengths $6 \mu \mathrm{m}$ and $4.28 \mu \mathrm{m}$. The dipoles are equal in width $(0.2 \mu \mathrm{m})$ and thickness $(0.1 \mu \mathrm{m})$. We have considered a dissipative transduction mechanism and the response is evaluated using the total power dissipated as Joule heating within the structure. In this configuration the 
resonant structure works as a distributed bolometer. ${ }^{5}$ Figure 1 shows the spectral response for three different states of polarization. We may check how the bandwidth is larger when both dipoles contribute to the total dissipation. This happens when the input radiation is linearly polarized and oriented at $45^{\circ}$ with respect to the orientations of the dipoles. As far as the $Y$ dipole is larger than the $X$ dipole, the dissipated power is larger and centered at a lower frequency.

This selective behavior can be better explained when calculating the spatial maps of the electric field and power dissipation. Figure 2 shows these maps for the three orientations of the incoming electric field already plotted in Fig. 1. The charge and current distribution can be extracted from the field maps. The analysis of the electric field distribution in terms of the orientation of the incoming field can be used to modify the far-field pattern or to enhance the results from microscopic techniques. ${ }^{15}$
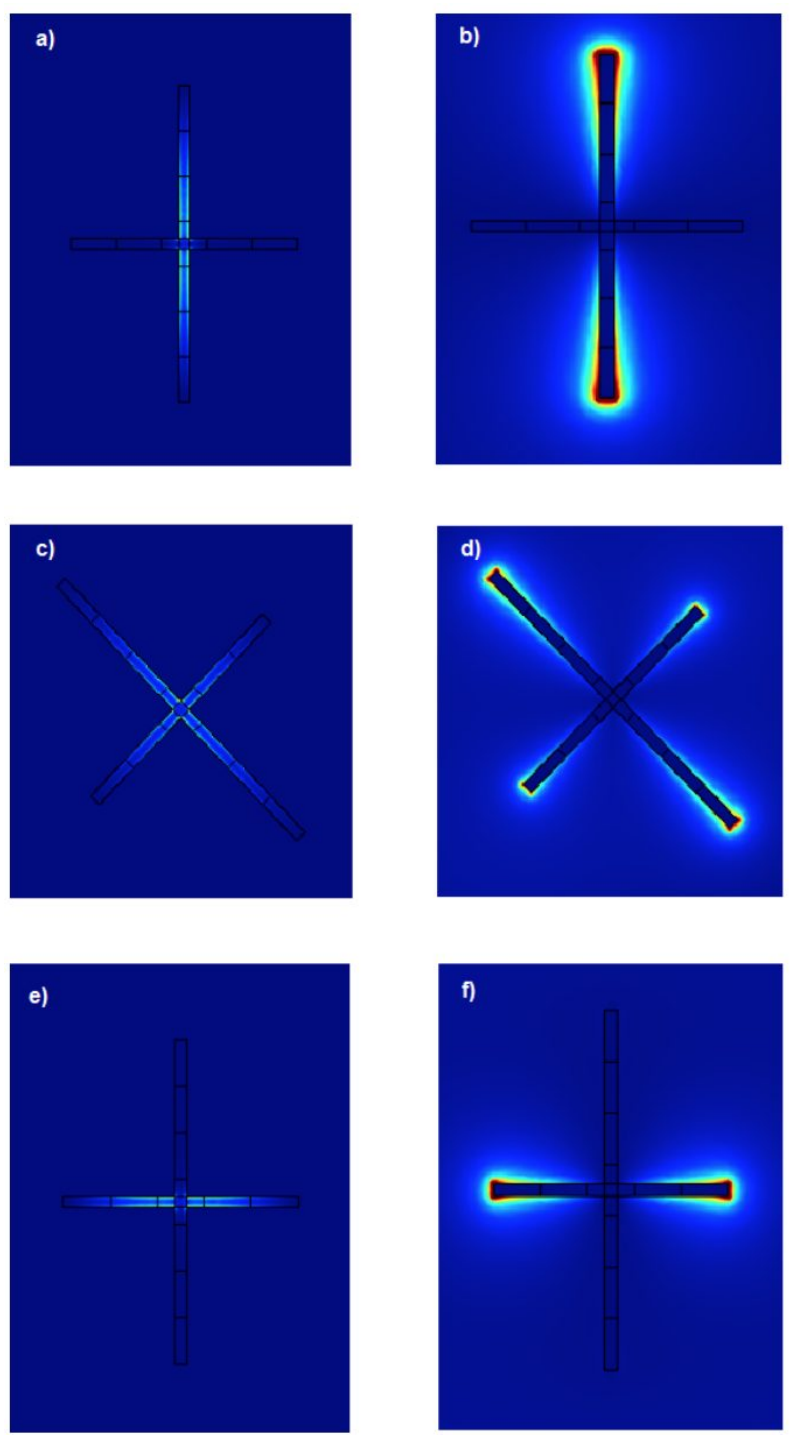

Figure 2. The left column shows the dissipated power map at different locations of the structure, and the right column represent the distribution of the electric field. Each map is calculated at the maximum response in frequency an polarization state. Plots a), b), c), and d) are for vertical polarization. Plots e) and f) are for horizontal polarization. 


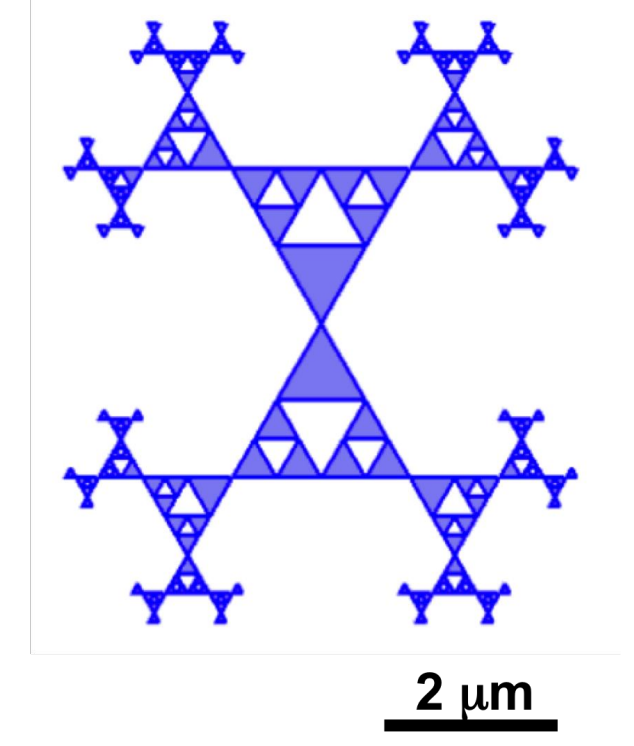

Figure 3. Fractal geometry based on a bow-tie antenna. This structure is expanded until fourth order fractal.

\subsection{Fractal antennas}

Fractal antennas are characterized by a self-similarity relation repeated at successive spatial scales. In this case, the resonances are located at different frequencies without changing the polarization state of the incoming wavefront. This fact has been used to design multispectra arrangements. In this section we analyze a modification of a bow-tie antenna that becomes a fractal antenna quite similar to the Sierpinski triangle already demostrated at lower frequencies. ${ }^{16}$ In this contribution we have studied the fractal structure shown in figure 3 . The central bow-tie has a diagonal length of $4 \mu \mathrm{m}$. The spectral behavior of the fractal antenna is given in Fig. 4. The incident wavefront is a linearly polarized plane wave having an electric field of $110 \mathrm{~V} / \mathrm{m}$. This plot shows a multiband behavior due to the fractal geometry. Three maxima are clearly seen in this spectrum. From the maps presented in Fig 5 we can relate them with the current modes excited within the structure. In this figure we have represented the dissipated power (left) and the electric field (right) generated when a given irradiance illuminates the fractal antenna. As far as the current density, $J$, and electric field, $E$, are proportional through electric conductivity $\sigma$, the map of the electric field can be also taken as the current density map when considering the distribution at the metal structure. Actually, the first maximum appears at a frequency lower than it should be expected for the unmodified bow-tie central geometry. This happens because, in the fractal bow-tie, the current density mode at this frequency travels along the central, largest diagonal, and extends towards adjacent fractal satellites. The second maximum appears closer to the resonant frequency of the basic unmodified bow-tie antenna, but the maps show how the maximum dissipation appears at the satellite fractals. The third maximum is related with current modes located at those portions of the fractal antenna of higher order.

From Fig. 5, we may check that the first mode dissipate a larger amount of power in the central portion of the fractal structures meanwhile the second and third maxima frequencies are dissipating at the higher-order fractal satellites. On the other hand, these fractal structures produce a field enhancement around the finer details (see the strong field enhancement around the metallic resonant structure)

Joule heat dissipation appears at those locations where currents flow. Therefore, the map of these currents is of great interest when considering the location of transduction elements or signal extraction lines. Figure 6 show the distribution of currents along the structure for the first and second maximum of figure 4 . We may see that second maximum at $32.5 \mathrm{THz}$ does not include contributions from currents at the large central fractal structure. Meanwhile, the central bow tie is the responsible for the appearance of the maximum response at $12.5 \mathrm{THz}$. In these previous cases, the orientation of the electric currents follows the vertical orientation of the incident electric field. In order to reinforce the selectivity of the antenna, and to relate it with the current paths, we also present 


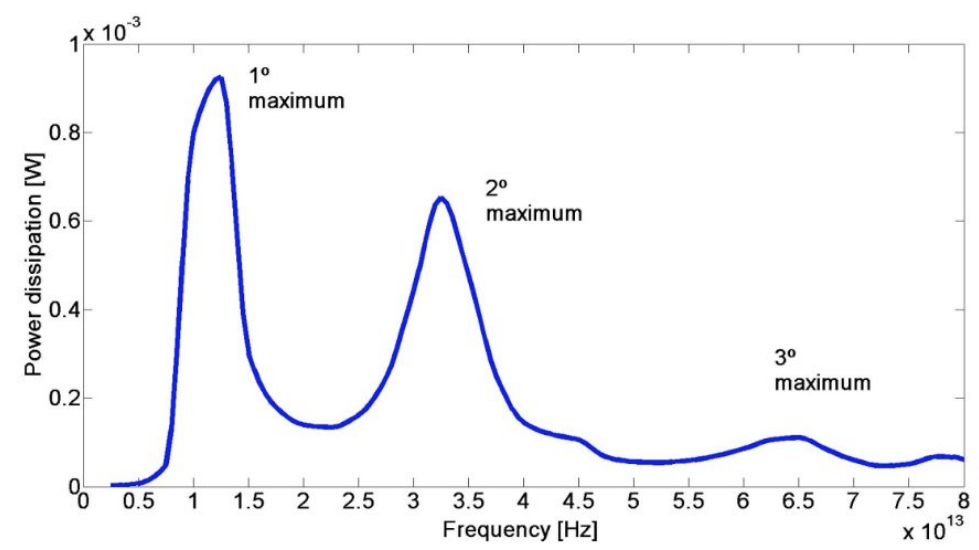

Figure 4. Spectral response of the fractal bow-tie presented in Fig. 3. The incidence is modeled as a plane wave having a constant electric field value of $110 \mathrm{~V} / \mathrm{m}$.
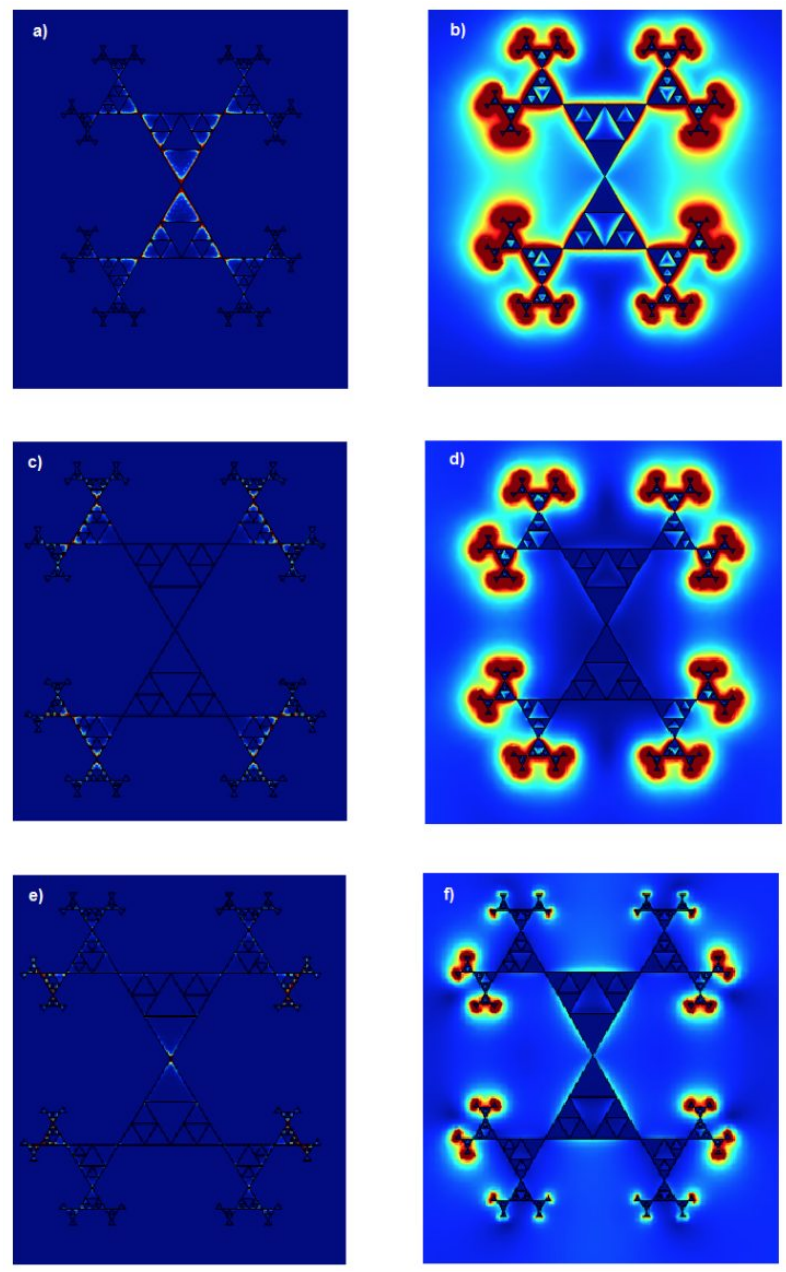

Figure 5. The left column represents the power dissipated by the fractal antennas, and the right column represents the electric field. The first row is for the first, largest, maximum of Fig. 4, the central row is for the second maximum, and the row at the bottom is for the third maximum. The polarization state is a linearly polarized light along $Y$ (vertical) direction. 
the current maps when polarization is at $45^{\circ}$ and frequency is $16.2 \mathrm{THz}$. Again, for this oblique polarizaton, the current paths are located where they can develop better for the given frequency and polarization.

Freq $1.25 \mathrm{E} 13 \mathrm{~Hz}$

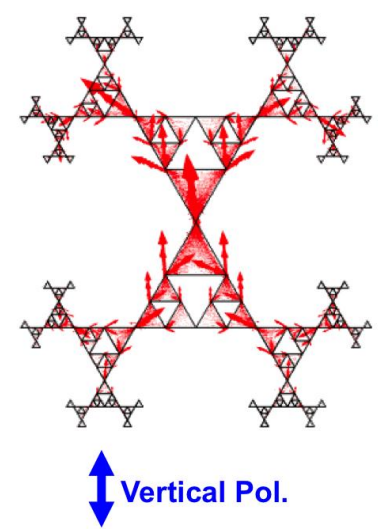

Freq $3.25 \mathrm{e} 13 \mathrm{~Hz}$

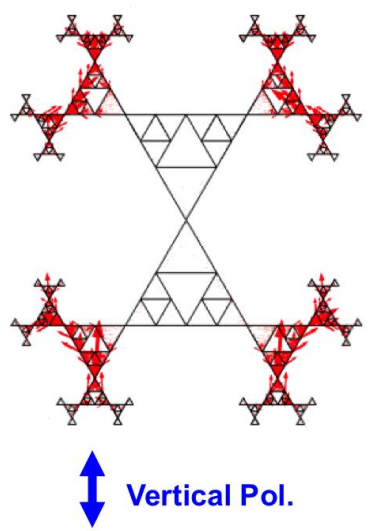

Freq. $1.62 \mathrm{E} 13 \mathrm{~Hz}$



Figure 6. Spatial distributions of currents for the first and second spectral maxima (left and center). In these cases the incident field is oriented along the vertical direction. The map at the right corresponds with the current distributions when the incident field is linearly polarized at $45 \circ$.

\section{CONCLUSIONS}

When using compound geometries combining dipoles of different lengths, it is possible to expand the spectral response by changing the state of polarization of the incoming wavefront. This type of different-length orthogonal dipoles can be of use for spectral and polarization selectivity. Fractal geometries allows an alternative way to create truly multispectra antennas. The response is linked with the basic geometry used to expand the fractal. The proposed fractal antennas are given from a variation of Sierpinski's triangle adapted to a bow-tie antenna. The computational electromagnetic simulations link the maxima in the spectrum with the current modes built in the fractal structure, according with the orientation of the electric vector of the incident wave.

Summarizing the findings of this contribution, we could visualize using computational electromagnetism tools a clear link between the geometry and the useful magnitudes in optical antennas. If antennas are used for detecting light, power dissipation and current at the feed points are the best parameters to monitor. In case optical antennas are used for local field enhancement, compound and fractal structures allows selectivity both in polarization and frequency to spatially locate the origin of the given response. An open problem that need to be addressed before fractal and compound antennas are used for light detection, is the location and shape of the signal extraction lines and the definition of the transduction mechanism.

\section{ACKNOWLEDGMENTS}

This work has been supported by the Ministerio de Economia y Competitividad of Spain through projects TEC2013-40442 and TEC2012-38901-C02-01. F. J. Gonzalez would like to acknowledge support by project 32 of Centro Mexicano de Innovación en Energía Solar from Fondo Sectorial CONACYT-Secretaría de EnergíaSustentabilidad Energética and by the National Laboratory program from CONACYT through the Terahertz Science and Technology National Lab (LANCYTT) 


\section{REFERENCES}

[1] Bharadwaj, P., Deutsch, B., and Novotny, L., "Optical antennas," Adv. Opt. Photon 1, 438-483 (2009).

[2] Novotny, L. and van Hulst, N., "Antennas for light," Nature Photonics 5, 83-90 (2011).

[3] Alda, J., Rico-García, J. M., López-Alonso, J. M., and Boreman, G., "Optical antennas for nano-photonic applications," Nanotechnology 16(5), S230 (2005).

[4] Fumeaux, C., Herrmann, W., Kneubühl, F. K., and Rothouizen, H., "Nanometer thin-film ni-nio-ni diodes for detection and mixing of 30 thz radiation," Infrared Physics and Technology 39, 123-183 (1998).

[5] Cuadrado, A., Alda, J., and Gonzalez, F. J., "Distributed bolometric effect in optical antennas and resonant structures," Journal of Nanophotonics 6(1), 063512-1-12 (2012).

[6] Cuadrado, A., Briones, E., Gonzalez, F. J., and Alda, J., "Polarimetric pixel using seebeck nanoantennas," Optics Express 22(11), 13835-13845 (2014).

[7] Novotny, L., "Effective wavelength scaling for optical antennas," Physical Review Letters 98, 266802-4 (2007).

[8] Silva-Lopez, M., Cuadrado, A., Llombart, N., and Alda, J., "Antenna array connections for efficiente performance of distributed mricrobolometers in the ir," Opt. Express 21(9), 10867-10877 (2013).

[9] Cuadrado, A., Alda, J., and Gonzalez, F. J., "Multihpysics simulation for the optimization of optical nanoantennas working as distributed bolometers in the infrared," Journal of Nanophotonics 7, 07093 (2013).

[10] Cuadrado, A., Silva-Lopez, M., Gonzalez, F. J., and Alda, J., "Robustness of antenna-coupled distributed bolometers," Optics Letters 38(19), 3784-3787 (2013).

[11] Gottheim, S., Zhang, H., A., G., and Halas, N., "Fractal nanoparticle plasmonics: the cayley tree," ACS Nano 9(3), 3284-3292 (2015).

[12] Volpe, G., G., V., and Quidant, R., "Fractal plasmonics: subdiffraction focusing and broadband spectral response by a sierpinski nanocarpet," Optics Express 19(4), 3612-3618 (2011).

[13] Simon, J. and Gonzalez, F. J., "Nanoantennas for polarisation division multiplexing," Electronics Letters 47(2), 120-121 (2011).

[14] Krenz, P., Alda, J., and Boreman, G., "Orthogonal dipole antenna," Infrared Physics and Technology 51(4), 340-343 (2008).

[15] Crozier, K. B., "Optical antennas: resonators for local field enhancement," Journal of Applied Physics 94, 4633-4641 (2003).

[16] Puente-Baliarda, C., Romeu, J., Pous, R., and Cardama, A., "On the behavior of the sierpinski multiband fractal antenna," IEEE Transaction on Antennas and Propagation 46(4), 517-524 (1998). 